\title{
George Sand journaliste, sous la direction de Marie- Ève Thèrenty
}

\section{Michel Arrous}

\section{(2) OpenEdition}

\section{Journals}

Édition électronique

URL : http://journals.openedition.org/studifrancesi/3797

DOI : 10.4000/studifrancesi.3797

ISSN : 2427-5856

Éditeur

Rosenberg \& Sellier

\section{Édition imprimée}

Date de publication : 1 décembre 2012

Pagination : 590-592

ISSN : 0039-2944

\section{Référence électronique}

Michel Arrous, « George Sand journaliste, sous la direction de Marie-Ėve Thèrenty », Studi Francesi [En ligne], 168 (LVI | III) | 2012, mis en ligne le 30 novembre 2015, consulté le 05 mars 2021. URL : http:// journals.openedition.org/studifrancesi/3797 ; DOI : https://doi.org/10.4000/studifrancesi.3797

Ce document a été généré automatiquement le 5 mars 2021.

\section{cc) (†) $९$}

Studi Francesi è distribuita con Licenza Creative Commons Attribuzione - Non commerciale - Non opere derivate 4.0 Internazionale. 


\title{
George Sand journaliste, sous la direction de Marie-Ève Thèrenty
}

\author{
Michel Arrous
}

\section{RÉFÉRENCE}

AA. VV., George Sand journaliste, sous la direction de Marie-Ève THÈRENTY, Presses de l’Université de Saint-Étienne, 2011, pp. 298.

1 Dans le renouveau des études sandiennes, on distinguera l'année 2011 avec la publication d'un George Sand critique (voir notre recension, «Studi francesi», n, 167) que vient enrichir ce George Sand journaliste. L'œuvre d'une journaliste prolixe qui occupa une place à part dans l'histoire du journalisme méritait un second volet. En présentant ce recueil (pp.7-20), Marie-Ève THÉRENTY souligne l'ampleur d'une carrière journalistique - près de 400 articles - et l'importance que lui a attachée George Sand puisqu'elle a recueilli beaucoup d'entre eux en volumes. Formée à l'école du «Figaro», elle accède dès 1832 à cet empyrée littéraire qu'était la «Revue des deux mondes», avec laquelle elle rompra en 1841 et qu'elle ne retrouvera qu'en 1858. Entre temps, tout en se montrant plutôt méfiante à l'égard du système médiatique, elle a vu dans la presse un outil d'émancipation pour le peuple, d'où la création de la «Revue indépendante» et de «L'Éclaireur de l'Indre». Les quatre parties de ce vrai livre («Les postures de l'engagement journalistique», «Poétiques journalistiques», «Feuilletons et fictions», «George Sand face à la presse») donnent une vue fort précise de son univers journalistique.

2 Anne MC CALl (La poétique du mépris: une stratégie problématique de la lettre ouverte, pp. 23-35), aborde les Lettres d'un voyageur par leur ambiguïté générique - mais le leurre de la lettre ouverte ne trompe guère! - et surtout cherche à comprendre la stratégie mise en œuvre avec l'exemple le plus contestable de l'ensemble, «Le Prince»[de Talleyrand], où se «manifeste une des visées essentielles des lettres ouvertes, l'expression directe du blâme». Il y a chez Sand une évidente inclination à la polémique 
et à l'accusation qu'on retrouve dans l'épisode de Fanchette. Les Lettres à Marcie, publiées en 1837 dans «Le Monde» de Lamennais, ayant déjà fait l'objet de plusieurs études, Nigel HARKNESS a voulu traiter des Problèmes d'énonciation et de réception dans les "Lettres à Marcie" (pp. 37-49), afin de les resituer dans leur contexte journalistique et d'analyser leur «écriture féminine et oppositionnelle». Ces Lettres furent à la fois la cible des fouriéristes de «La Phalange» qui virent en leur auteur le porte-parole de Lamennais, et victimes de la censure du «Monde» qui en interrompit la publication. Autre exemple de la magistrature de la parole doublée d'un sens étonnant de la controverse: «Fanchette, lettre de Blaise Bonnin à Claude Germain», fait divers berrichon traité dans la «Revue indépendante» du 25 octobre 1843, et la polémique qui s'ensuivit avec le procureur du roi de La Châtre. Dans cette plaidoirie en faveur des droits humains, Catherine NESCI (Communication et espace public chez George Sand, pp. 51-63) met l'accent sur l'habileté et l'inventivité de Sand. Statistiques à l'appui, Thomas LouÉ analyse la seconde phase de la relation de Sand avec Buloz: Quelques remarques sur un auteur en institution (1858-1876) (pp. 65-82). On peut parler d'un retour au bercail et d'une nouvelle expérience éditoriale faite de relations «toujours délicates et complexes», pendant ces dix-huit années où la «Revue des deux mondes» publia quarante-cinq œuvres de Sand.

On revient à l'affaire Fanchette avec Claudine GROSSIR, cette fois pour illustrer l'attention et la fidélité aux faits, mais aussi l'évolution esthétique de Sand qui cherche toujours à s'affranchir des contraintes génériques (George Sand journaliste: l'invention de l'actualité, pp. 85-95). Autre nouveauté dans cette création journalistique avec le récit de voyage qui, tout en s'inscrivant dans une longue tradition, oscille entre le privé et le public. L'affectivité se manifestant davantage dans le premier ensemble que dans le second, selon Roland LE HUENEN (Le voyage sandien dans la «Revue des deux mondes»: "Lettres d'un voyageur" et "Un Hiver à Majorque", pp.97-109). Catherine MAsson présente un exemple fort rare de la poétique journalistique avec "Le Père Va-tout-seul": théâtralisation de l'article politique sandien (pp.111-122): publié en 1845 par le militant républicain Pagnerre, cet article peut se lire comme une pièce de théâtre en un acte, un exemplum théâtralisé dont l'intention didactique est évidente. Considérant George Sand critique littéraire (pp.123-137), autrement dit l'ensemble de sa production critique, Christine PLANTÉ met en évidence sa cohérence: «La critique est une législation ou un enseignement», écrit Sand dans son article de 1864 sur le William Shakespeare de Hugo, mais à la mission didactique et pédagogique s'ajoute l'impératif de solidarité esthétique avec les écrivains, ou du moins avec certains d'entre eux, car Sand pratique une critique de sympathie, une critique des beautés qui ne s'interdit pas «un peu d'examen préalable», comme elle le dit à propos de L'Oiseau de Michelet. On s'étonne parfois que les arts figuratifs soient le seul domaine où Sand ne se soit pas impliquée véritablement; cependant les contributions, qui se recoupent d'ailleurs, de MarieHélène GIRARD, dans Critique d'art / George Sand dans la mêlée (pp. 139-157) et de François KERLOUÉGAN, «Un grand effet obtenu par les moyens les plus simples»: George Sand critique d'art (pp. 159-173), corrigent cette impression à partir des articles écrits lors de l'Exposition de 1855, qu'il s'agisse de Delacroix, qu'elle admire, d'Ingres sur lequel elle fait des réserves tout en louant le Vœu de Louis XIII gravé par Calamata, ou de Courbet, que Champfleury défend auprès d'elle, à l'occasion de l'exposition privée de 1855 qu'elle a boudée. Contrairement à ce que pensait Champfleury, la cause du réalisme n'était pas la sienne! Sand a donc peu écrit sur la peinture, mais de ce peu on tirera, plutôt qu'un 
manifeste esthétique en bonne et due forme, un souci de vérité et de simplicité. Gabrielle MELISON a eu la curiosité de lire la douzaine d'obituaires en forme d'hommage que Sand a rédigés (Les articles nécrologiques de George Sand, pp. 175-187). L'intérêt de ces écrits rédigés en l'honneur de personnalités dont la notoriété était parfois locale réside dans l'habileté de Sand à détourner les codes d'un genre pas encore stabilisé, comme on peut le voir dans la lettre sur la mort du comte d'Orsay («La Presse», 11 août 1852), ou dans le portrait en action de Latouche («Le Siècle», 18-20 juillet 1851). Dans cette écriture pour autrui se lit aussi une écriture de soi ou le désir d'appréhender sa propre mort.

4 Dans la troisième partie, trois cas permettent d'illustrer la stratégie médiatique de G. Sand. En retraçant La publication en feuilletons d'une autobiographie: "Histoire de ma vie" de George Sand dans «La Presse» (de la scandaleuse à la sainte) (pp. 191-201), Lise DUMASY rappelle la volonté de contrôle et la tactique déceptive de l'autobiographe qui a toujours et farouchement gardé secrète sa vie privée, sans doute par souci des bienséances, mais aussi parce qu'elle a pu, après la mort de Musset, répondre aux attaques de façon détournée par voie de roman (Elle et Lui, dans la «Revue des deux mondes», janvier-mars 1859). Daniel DesormeAux traite Le cas des "Beaux Messieurs de Bois-Doré" (pp. 203-217): Sand, qui ne se reconnaissait pas le talent de Dumas ou de Sue, va souvent à contre-courant des idées sur le roman-feuilleton populaire. Alors que Zola a affirmé que sa consœur se lançait dans l'écriture sans plan préconçu, l'étude de la genèse et de la composition de cette œuvre hybride (reportage historique et fiction romanesque, parodie du roman-feuilleton et critique sociale) prouve la mise en œuvre d'une stratégie contre le roman-feuilleton, mais bien sûr pour d'autres raisons que celles qui ont déclenché la polémique en 1836. Démonstration confirmée par Olivier BARA (“Lucrezia Floriani” de George Sand: art et stratégie de l'«anti-feuilleton», pp. 219-233), qui, avec l'exemple de cette «fracassante manifestation d'indépendance face aux lecteurs et aux directeurs de presse», illustre la volonté de Sand d'aller contre les dérives et les inflations du genre mis à la mode et exploité par Gautier, Dumas, Méry, Sue, Soulié («Ah! mon ami, quelles savates!», à Delacroix, 28 septembre 1845).

Le journalisme «autocentré» de Sand peut aussi s'évaluer par le biais de son attitude face à la presse. Après B. Tillier (1993), L. Czyba (1995) et B. Diaz (1998), Michèle FONTANA examine le traitement de l'image de l'écrivain dans la «fièvre caricaturale» qui s'est emparée de la France après Juillet (George Sand fecit soi-même: George Sand face à sa caricature, pp. 237-257, avec quatorze illustrations). Le "super bas-bleu» a inspiré Daumier («La Caricature», 18 juillet 1844), mais il a été aussi une victime de choix pour Granville, Bertall ou Nadar. Amusée et parfois agacée, il n'en reste pas moins que «Sand a gagné la bataille de l'image face au public» et que s'est imposé le bon portrait en «institutrice du peuple». Des diverses époques journalistiques, Béatrice DiDIER propose une lecture synoptique des préfaces que Sand a été amenée à rédiger pour ses đEuvres, bien loin d'être complètes en 1842, puis en 1851 et en 1875 (Les préfaces des "Eeuvres complètes": duel et réconciliation avec la presse, pp. 259-265). L'examen des brouillons, par A. Szabó, a montré que le point de vue de Sand a évolué: d'abord sur la défensive, voire toute à l'offensive et à la critique de la critique, la romancière élargit le regard sur son œuvre et par rapport à l'évolution esthétique $d u$ XIX siècle. Restent à évoquer, pour mieux la situer, ses très rares contemporaines qui ont pu faire du journalisme politique comme les hommes sous le Second Empire. Alice PRIM s'y est employée (Les «consœurs» de George Sand: divers usages de la presse par des femmes politiquement engagées (1848-1870), 
pp. 267-277). Toutes ont dû déjouer les règles établies et trouver des compromis pour se faire entendre. Sand apparaît bien, dans ce domaine, comme "une sorte d'hapax». Il faudra attendre la fin du siècle pour retrouver, avec Séverine, le même phénomène.

6 Alors que le geste journalistique chez Sand a longtemps été sous-estimé et même occulté, cet ouvrage incite à le redécouvrir et à apprécier toute son importance. 